Reprod. Nutr. Dévelop., 1988, 28 Suppl. $\mathrm{n}^{\circ} 1,33-58$

\title{
La modélisation de la digestion dans le rumen
}

\author{
D. SAUVANT
}

Station de Nutrition et Alimentation, I.N.R.A./I.N.A.-P.G., 16, rue Claude-Bernard, 75231 Paris Cedex 05.

\section{Summary. Modelling of rumen digestion.}

The ruminal ecosystem illustrates the main concepts of system analysis. Due to its structure and function complexity and to its determinant role in ruminant nutrition and feeding, the reticulo-rumen has been the target of modelling works. The proposed models have been static or dynamic. The later have known a recent development but the former have until now largely contributed to the knowledge progress. The complexity of dynamic models is proportional to the number of biological mechanisms taken into account. The simplest models describe for instance a degradation or a transit elementary process. The aggregated models take simultaneously into account 2 to 4 processes while the general models aim at describing the digestive behaviour of the ruminal ecosystem by integrating its main functions. These mechanistic models have been studied for about 20 years. They are on the whole satisfactory but they are not sufficiently able to describe some diet differences and some variations in the dynamic properties of feed particles.

\section{Introduction.}

Une meilleure compréhension et la maîtrise de la digestion réticulo-ruminale ont constitué un des grands défis des recherches zootechniques, si bien que d'innombrables publications lui ont été consacrées. II y a plus de 20 ans, Hungate (1966) a utilisé près de 2500 références pour réaliser le seul ouvrage d'ensemble sur le sujet. En outre, une exploration de la bibliographie révèle que depuis 1973 une moyenne de 465 articles ont été annuellement publiés avec le rumen parmi les mots clefs. Cette masse informationnelle et son degré de dispersion soulèvent un problème de synthèse et d'intégration dans un ensemble de connaissances finalisées. En outre, si la tendance à la réduction du nombre de publications observées depuis 1980 se confirme, il conviendra de choisir encore plus précisément chaque thématique de recherche de manière à apporter le maximum de « connaissance ajoutée » par rapport au capital informationnel déjà accumulé.

Dans le domaine de la digestion ruminale, la modélisation a été perçue depuis une trentaine d'années comme un moyen d'intégrer les données nouvelles en un ensemble synthétique et facilement utilisable en vue de l'analyse de besoins en recherche ou des applications. Les précurseurs ont vraisemblablement été Blaxter, 
McGraham et Wainman (1956) et surtout Hungate (1966) qui se sont focalisés sur la modélisation de certains phénomènes de transit. A leur suite, quelques équipes se sont consacrées à la mise au point et à l'amélioration de modèles capables de traduire des aspects partiels ou globaux du fonctionnement du réticulo-rumen. La présente revue a pour but de faire une analyse critique de ces propositions pour essayer de dégager les voies d'investigations pour lesquelles la modélisation présente le plus d'intérêt.

\section{L'écosystème ruminal considéré à travers les concepts de la systémi- que.}

Les concepts de la théorie des systèmes (Walliser, 1977; Von Bertalanffy, 1979 ; Durand, 1983) se retrouvent dans les aspects connus du fonctionnement du réticulo-rumen. La paroi ruminale illustre bien la notion de frontière d'un système ; les ingesta et les passages de substances en provenance de l'organisme représentent les flux d'entrée alors que les flux de sortie correspondent aux nutriments absorbés et aux passages des digesta dans la suite du tube digestif. Les éléments du système ruminal dépendent du thème d'étude considéré, il peut s'agir:

- des principales fractions biochimiques ou anatomiques des aliments et des substances issues de leur dégradation ;

- des éléments $(\mathrm{C}, \mathrm{H}, \mathrm{O}, \mathrm{N}, \ldots)$ constitutifs de la biomasse contenue dans le rumen (Reichl et Baldwin, 1976) ;

- de la micropopulation ruminale considérée comme un tout ou bien répartie en sous-populations distinguées par des critères métaboliques (Baldwin, Lucas et Cabrera, 1970) ou de fixation aux particules (Cheng et Costerton, 1980; Czerkawski, 1984);

- des particules alimentaires distinguées par classe de taille (Ulyatt, Baldwin et Koong, 1976 ; Faichney, 1984).

Les relations qui associent les éléments constitutifs de tout système représentent par exemple pour le réticulo-rumen :

- des transferts de matières entre le substrat et la biomasse microbienne ou bien entre celle-ci et le milieu ruminal (et réciproquement) :

- des transferts de métabolites d'un type de micro-organisme à un autre, la prédation des bactéries par les protozoaires;

- des transferts de particules d'un compartiment de classe de taille à l'autre.

La présence de structures ayant fonction de réservoir permet par exemple d'assurer, par le biais de délais, une certaine régularité et optimisation du fonctionnement du rumen dans le temps:

- la séquestration des particules de taille importante de manière à les soumettre pendant plus longtemps à l'action physique de la rumination et enzymatique des microorganismes :

- le stockage temporaire, en phase post-prandiale, de polyosides microbiens (Jouany et Thivend, 1972 ; Craig et al., 1984);

- la séquestration relative de $\mathrm{NH}_{3}$ libéré après un repas grâce à la baisse simultanée du $\mathrm{pH}$ 
Le concept de centre de décision est bien illustré par un certain nombre de régulations, l'exemple bien connu étant l'influence de la fibrosité de la ration et de la distension ruminale sur les phénomènes de rumination et de salivation. De ce fait, l'écosystème ruminal contient en partie les éléments de sa propre finalité, par contre, il ne possède pas de propriétés de mémorisation ou d'imagination connues pour d'autres systèmes.

Le fait que le comportement digestif et fermentaire global de la micropopulation ruminale ne peut être prévu par la simple addition de ces mêmes propriétés mesurées au niveau de chaque espèce étudiée de façon séparée (cf. Gouet et al., 1986) illustre le principe de totalité d'un système. Or, pour d'évidentes raisons de moyens d'investigation, les recherches sur la digestion dans le rumen se sont largement conformées à une approche cartésienne: l'écosystème ruminal a été ainsi divisé en "parcelles " de sous-difficultés accessibles aux investigations, si bien que les différents processus digestifs ont été étudiés par fragment et d'une façon relativement isolée les uns des autres. La connaissance globale du fonctionnement du réticulo-rumen tend, de ce fait, à être établie à partir de l'addition de celle des phénomènes élémentaires. Ce type d'approche a peut-être favorisé la connaissance sur les caractéristiques " sommatives " du réticulo-rumen alors que ses caractéristiques constitutives, associées au principe de totalité, plus difficile à appréhender expérimentalement, présentent peut-être le plus d'intérêt agronomique (s.l.).

Un des objectifs de l'approche systémique est de privilégier la connaissance globale du fonctionnement du rumen, c'est-à-dire en particulier de ne pas négliger ses caractéristiques constitutives. Une telle démarche suppose notamment de travailler en permanence avec un modèle global (voir plus loin) de manière à pouvoir, d'une part, mieux détecter les mécanismes sur lesquels focaliser les recherches et, d'autre part, mieux appréhender le rôle de chaque mécanisme considéré sur le fonctionnement de l'ensemble.

\section{La modélisation de la digestion ruminale.}

Le terme de modèle regroupe en fait une multitude de significations et la définition rappelée par Spedding (1988) convient à l'usage qui peut en être fait dans le cas du rumen:

«Abstraction et simplification du monde réel capable d'intégrer les principales interactions et le comportement du système étudié, apte à être manipulé en vue de prévoir les conséquences de la modification d'un (ou plusieurs) déterminant sur le comportement du système. " L'abstraction et la simplification évoquées revêtent fréquemment, mais pas obligatoirement (exemple : le Rusitec), un aspect mathématique.

Les étapes de l'élaboration d'un modèle ont été résumées par Baldwin et Koong (1979). Différents types de modèles peuvent être définis en fonction de leurs principales caractéristiques; présentement, une distinction générale sera faite entre les modèles statiques et dynamiques. A l'intérieur de ces 2 groupes 
coexistent : 1) des modèles empiriques qui ajustent des données expérimentales et décrivent le comportement de systèmes sans en connaître les rouages internes (= boîte noire), et 2) des modèles explicatifs ou mécanistes qui ont pour but de simuler le comportement du système en intégrant le fonctionnement de ses éléments. En outre, des modèles théoriques ont parfois été proposés et les recherches consécutives ont eu pour objet de vérifier leur bien-fondé et de déterminer les valeurs des paramètres.

\section{Les modèles statiques.}

Ces modèles ne font pas intervenir le temps ce qui revient à supposer que le, ou les, processus considéré(s) se maintienne(nt) au sein d'un état stationnaire «moyen » et, en particulier, que les apports de substrat sont réalisés d'une façon continue. Ils sont en outre dans la plupart des cas de type empirique et les lois obtenues décrivent le plus souvent des mécanismes biologiques à caractère synthétique. Ces lois prennent, par contre, un rôle explicatif à partir du moment où le système de référence correspond à un ensemble plus large, le réticulo-rumen ou l'organisme animal entier par exemple (Sauvant, 1988).

Les premières lois du fonctionnement du rumen obtenues traduisaient les résultats d'une seule expérimentation, leur domaine d'application était de ce fait très restreint. Depuis une quinzaine d'années, la tendance est de pratiquer ce type d'analyses, sur des bases de données regroupant les résultats expérimentaux obtenus par plusieurs équipes. Moyennant un certain nombre de précautions statistiques, cette démarche permet d'obtenir des lois possédant une meilleure universalité. De telles démarches ont, par exemple, été effectuées dans le cadre de la mise au point de certains nouveaux systèmes d'unités d'alimentation azotée du ruminant (Roy et al., 1977 ; Jarrige, Journet et Vérité, 1978, etc...).

La progression des connaissances à partir d'un modèle théorique est illustrée par la loi décrivant a priori la croissance microbienne en fonction de la disponibilité en ATP (Pirt, 1965; Stouthamer et Bettenhaussen, 1973). De nombreuses études expérimentales ont cherché à valider ce modèle et à déterminer les valeurs de ses paramètres pour des biotopes anaérobies ( $c f$. Belaich, 1986) ou pour les bactéries du rumen (cf. Demeyer et Van Nevel, 1986). Les équations stœchiométriques de la production des $A G V$, de $N_{3}$, de gaz... à partir des quantités de substrat dégradées qui ont été utilisées au sein de plusieurs modèles globaux (voir plus loin) avaient également à l'origine un caractère théorique dans la mesure où elles avaient été largement établies à partir de la connaissance des voies métaboliques étudiées avec d'autres micro-organismes que ceux du rumen, ainsi que sur des «présomptions logiques » (Baldwin, Lucas et Cabrera, 1970). Ultérieurement, Murphy, Baldwin et Koong (1982) se sont appuyés sur une large base de données expérimentales (108 régimes distribués à 511 animaux) pour obtenir des estimations empiriques des coefficients des équations stœchiométriques des fermentations des glucides (soluble, amidon, hémicellulose, cellulose) et des protéines. Les résultats ont révélé une bonne cohérence entre les approches théoriques et empiriques. Cependant, il s'est avéré qu'une distinction doit être faite entre les régimes à base de fourrages et ceux contenant des aliments concentrés. 
Ces quelques exemples montrent qu'il n'existe pas une règle générale d'application des modèles statiques pour progresser dans les connaissances.

\section{Les modèles dynamiques.}

Leur propriété essentielle est d'intégrer le temps; ils permettent de ce fait de décrire l'évolution des caractéristiques du système ruminal. Une distinction est faite selon que le modèle s'applique à un ou à plusieurs maillons de la digestion, ou bien qu'il a pour but de traduire l'ensemble du fonctionnement du réticulo-rumen.

\section{Les modèles partiels simples.}

Le transit des particules. - L'évolution temporelle de la concentration de particules marquées (oxyde de chrome, colorants, terres rares...) dans le rumen (Yt) a été depuis longtemps ajustée à un modèle de type exponentiel simple (Hungate, 1966). La qualité de tels ajustements est généralement satisfaisante et permet de dire que l'évacuation des particules se conforme à un processus exponentiel du $1^{\text {er }}$ ordre, le rumen étant assimilé à un compartiment unique et homogène :

$$
\mathrm{dYt} / \mathrm{dt}=-\mathrm{k} \cdot \mathrm{Yt} \Rightarrow \mathrm{Yt}=\mathrm{Yo}_{\mathrm{o}} \cdot \mathrm{e}^{-\mathrm{kt}}
$$

avec $Y_{0}=$ Concentration en particule à l'instant $\mathrm{t}=\mathrm{o}$

$k$ = Taux d'évacuation des particules par unité de temps.

Ce modèle suppose une diffusion instantanée du marqueur dans le compartiment ruminal ou bien s'applique à partir d'un état d'équilibre atteint après une infusion continue de particules marquées pendant une durée suffisante.

En réalité, il arrive que l'ajustement ne soit pas statistiquement satisfaisant, en raison par exemple du phénomène de comminution des particules, qui entraîne une variation du coefficient $k$ en fonction du temps:

$$
d Y t / d t=-k(t) \cdot Y t .
$$

La résolution de cette équation différentielle demande de connaître la fonction $k(t)$ ce qui n'a, à notre connaissance, jamais été publié. Par contre, des ajustements statistiquement satisfaisants ont été obtenus en utilisant un modèle à plusieurs compartiments, deux par exemple :

$$
Y_{t}=Y_{o}\left(p \cdot e^{-k 1 t}+q \cdot e^{-k 2 t}\right)
$$

$p$ et $q$ étant les proportions des 2 compartiments $(p+q=1)$ et $k 1$ et $k 2$ les taux d'écoulement hors des 2 compartiments. II n'a toutefois pas encore été possible de diviser, par cette voie, le système ruminal en sous-systèmes homogènes ayant une signification biologique.

L'ajustement de l'évolution du flux sortant des particules marquées placées à un instant $\mathrm{t}=\mathrm{o}$ dans le réticulo-rumen soulève plus de problèmes dans la mesure où le flux ne débute pas forcément à $t=0$, ce qui crée un temps de latence, puis augmente jusqu'à un maximum avant de diminuer. 
L'interprétation de telles courbes d'excrétion dont l'allure se retrouve à des parties plus distales du tube digestif, en particulier au niveau des fèces, suppose en général l'existence $d$ 'un système à 2 compartiments sans réversibilité :

$$
\mathrm{Y} 1 \mathrm{t} \longrightarrow(\mathrm{k} 1) \rightarrow \mathrm{Y} 2 \mathrm{t}-(\mathrm{k} 2) \rightarrow
$$

Y1t et Y2t correspondent aux quantités de particules présentes dans les compartiments 1 et 2 , $k 1$ et $k 2$ représentent les taux de sortie des particules de ces deux compartiments. Dans l'hypothèse où la sortie des particules est proportionnelle à leur concentration dans le compartiment considéré le fonctionnement de ce système peut être décrit par les deux équations différentielles suivantes:

$$
\begin{aligned}
& \mathrm{d} Y 1 t / d t=-k 1 \cdot Y 1 t \\
& d Y 2 t / d t=k 1 \cdot Y 1 t-k 2 \cdot Y 2 t .
\end{aligned}
$$

Ce système permet d'obtenir par intégration l'expression de l'évolution de la concentration en particules dans l'ensemble du système :

$$
Y(t)=\frac{Y 0}{k 2-k 1}\left(k 2 e^{-k 1 t}-k 1 e^{-k 2 t}\right)
$$

Plusieurs groupes de recherches se sont penchés sur le problème de la modélisation de telles courbes. Le modèle présenté ci-dessus a été proposé par Blaxter, McGraham et Wainman (1956); des adaptations de ce modèle ou l'emploi d'autres formes mathématiques ont notamment été proposées par Grovum et Williams (1973), Ellis, Matis et Lascano (1979), Dhanoa et al. (1985). Ces différents modèles souffrent d'un même inconvénient: les compartiments qu'ils définissent ne peuvent pas être précisément associés à des événements digestifs ou des compartiments anatomiques classiquement admis. En outre, il serait possible d'admettre une réversibilité entre certains compartiments et de tester cette hypothèse comme l'ont fait Jolivet, Laplace et Lebas (1975) dans l'étude du transit du lapin.

Une approche plus réaliste et porteuse de progrès de la modélisation des phénomènes de transit est permise par le marquage différentiel des particules selon plusieurs classes de taille (Faichney, 1984 ; Ellis et al., 1984 ; Poncet et Faichney, non publié). Ces données devraient permettre en effet d'élaborer des modèles ayant 6 à 8 compartiments pour mieux décrire la dynamique d'ensemble des particules et des populations microbiennes.

Le transit des micropopulations. - Les micropopulations ruminales peuvent être réparties selon la taille des particules alimentaires sur lesquelles elles adhèrent. Ainsi, Czerkawski (1984) et Owen et Goetsch (1984) proposent de discriminer 3 groupes de microorganismes $A, B, C$ attachés aux particules de tailles respectives $<200 \mu$, de 200 à $1200 \mu$ et $>1200 \mu$. Les organites de la classe $C$ sont séquestrés dans le rumen tandis que les autres passent dans la suite $\mathrm{du}$ tube digestif avec les taux $\mathrm{ka}$ et $\mathrm{kb}$ de leurs particules de fixation. Ce raisonnement peut se généraliser à une partition en $n$ compartiments, de proportion $\mathrm{Ci}$, de la micropopulation dont le taux moyen d'évacuation $(\mathrm{km})$ devient : 


$$
\mathrm{km}=\sum_{1}^{n} \mathrm{Ci} \cdot \mathrm{ki}
$$

ki étant le taux d'évacuation des particules alimentaires du compartiment i.

Les cinétiques de dégradation des aliments. - La dégradation ruminale des constituants des aliments conditionne largement l'efficacité de leur digestion ; elle a de ce fait été fréquemment l'objet d'une modélisation. Les modèles proposés sont de type empirique, ils ont été appliqués à la cinétique de la dégradation mais également à celle des quantités non dégradées. Ces ajustements doivent être pratiqués avec un ensemble de précautions statistiques qui sont décrites au chapitre $V$ de l'ouvrage de Tomassone, Lesquoy et Millier (1983).

Les modèles d'ajustement des cinétiques de dégradation. - L'évolution de la dégradation des substrats (Dt) résulte d'abord des caractéristiques de leur résistance à la dégradation microbienne. Si le substrat considéré est homogène et n'offre pas de résistance à la dégradation, la vitesse de dégradation à l'instant $\mathbf{t}$ $(\mathrm{dDt} / \mathrm{dt})$ est proportionnelle à la quantité déjà dégradée à ce moment :

$$
\mathrm{dDt} / \mathrm{dt}=\mathrm{C} \cdot \mathrm{Dt}
$$

ce qui aboutit, par intégration, à une cinétique correspondant à une exponentielle croissante qui cesse brutalement au moment de la disparition de la dernière particule de substrat. En réalité, le substrat offre presque toujours une certaine résistance qui se traduit, d'une part, par un ralentissement plus ou moins marqué de la vitesse de dégradation et, d'autre part, fréquemment par une dégradabilité potentielle, Dp, inférieure à $100 \%$. Une approche simple d'intégration de cette résistance suppose que la vitesse de dégradation est, à tout moment, proportionnelle à la fraction dégradable encore non dégradée :

$$
\mathrm{dDt} / \mathrm{dt}=\mathrm{c}(\mathrm{Dp}-\mathrm{Dt})
$$

ce qui aboutit par intégration à l'équation :

$$
\mathrm{Dt}=\mathrm{Dp}\left(1-\mathrm{e}^{-\mathrm{ct}}\right)
$$

dans laquelle c représente le taux de dégradation horaire $(0<\mathrm{c}<1)$ de la fraction $\mathrm{Dp}$. La valeur constante du paramètre $c$ suppose que la fraction dégradable $\mathrm{Dp}$ possède une résistance homogène vis-à-vis de l'attaque microbienne, ce qui n'est en fait pas toujours le cas. Une première façon de prendre en compte l'existence d'une résistance hétérogène consiste à déterminer au sein de la fraction $D p$ des sous-fractions homogènes ( $D p 1, D p 2 \ldots$ ) caractérisées par leur taux de dégradation propre $(\mathrm{c} 1, \mathrm{c} 2 \ldots)$. Cette hypothèse aboutit à une équation de dégradation du type

$$
D t=D p 1\left(1-e^{-c 1 t}\right)+D p 2\left(1-e^{-c 2 t}\right)+\ldots
$$

Si une de ces fractions, Dp1 par exemple, présente une valeur c1 très élevée, elle peut être considérée comme instantanément dégradable et l'expression précédente devient 


$$
D t=D p_{1}+D p_{2}\left(1-e^{-c 2 t}\right)+\ldots
$$

Ce type de modèle a été largement appliqué au cas de 2 fractions à la suite de la proposition de Ørskov et McDonald (1979); son succès s'explique, d'une part, par la facilité de déterminer ses 3 paramètres après transformation logarithmique et sans programme d'ajustement non linéaire, pratique non recommandable (Tomassone, Lesquoy et Millier, 1983) et, d'autre part, par sa capacité à tenir compte avec une expression simple du transit des particules (voir plus loin).

Ce modèle exponentiel du $1^{\text {er }}$ ordre présente l'inconvénient de n'intégrer que des phénomènes de résistance à la dégradation microbienne. Or, la dégradation débute souvent par une phase de latence, qui aboutit à une cinétique de type sigmoïde, ou une trajectoire linéaire si bien que l'estimation de Dp1 peut prendre une valeur négative. Différentes solutions ont été proposées pour intégrer l'existence d'une phase de latence. McDonald (1981), a proposé de n'appliquer le modèle de 2 fractions évoqué ci-dessus qu'à partir d'un temps $t=0$ égal au temps de latence. En fait, cette proposition est théorique et sans précision sur la méthode de détermination de $\mathrm{t}=0$ (par graphique ?). II semblerait plus rigoureux de chercher à travailler sur modèle à 4 paramètres calculant automatiquement le temps de latence $(t-$ to) :

$$
D t=D p 1+D p 2\left(1-e^{-c 2(t-t o)}\right) .
$$

II n'est en fait pas réaliste de supposer que le temps de latence est discret, c'est-à-dire que la cinétique de dégradation présente un «coude » à $t$ - to ; en effet, ce temps de latence correspond à une phase d'accélération de la dégradation (vitesse de dégradation proportionnelle à la quantité dégradée au même instant) progressivement remplacée par une résistance dominante, ce qui détermine une cinétique de dégradation présentant un point d'inflexion. Un moyen simple de décrire ce phénomène consiste à exprimer que la vitesse de dégradation est le produit de la quantité déjà dégradée $(D t)$ par une fonction de résistance $f(D t)$ :

$$
d D t / d t=f(D t) \cdot D t
$$

Par exemple, dans l'hypothèse où la fonction de résistance est, comme cela a déjà été évoqué, proportionnelle à la fraction dégradable non encore dégradée

$$
f(D t)=c(D p-D t)
$$

l'intégration de l'équation

$$
\mathrm{dDt} / \mathrm{dt}=\mathrm{c}(\mathrm{Dp}-\mathrm{Dt}) \cdot \mathrm{Dt}
$$

aboutit à une cinétique correspondant au modèle logistique fréquemment utilisé pour ajuster des courbes de croissance à allure sigmoïdale (Jolivet, 1983). Dans ce cas la vitesse de dégradation s'accroît dans un premier temps jusqu'à la valeur de dégradation de $\mathrm{Dp} / 2$ correspondant au point d'inflexion. Si on suppose par contre que la résistance suit une loi exponentielle décroissante

$$
f(D t)=a \cdot e^{-b t}
$$


l'expression de la vitesse de dégradation devient:

$$
d D t / d t=a \cdot e^{-b t} \cdot D t .
$$

L'intégration de cette équation aboutit au modèle de croissance de Gompertz (Jolivet, 1983) présentant un point d'inflexion lorsque la dégradation est égale à Dp/e. L'application de ce modèle (Sauvant et al., 1985 et non publié) à des cinétiques de dégradation in sacco de différents constituants des aliments permet d'obtenir, dans une partie des cas, des résultats bien plus satisfaisants qu'avec l'équation (1).

Ces deux exemples de modèle de croissance sont restrictifs dans la mesure où l'ordonnée du point d'inflexion est fixée. Il est possible d'utiliser des hypothèses de fonction de résistance plus complexe de manière à pouvoir paramétrer cette ordonnée (Richard, 1959).

Les modèles d'ajustement des quantités résiduelles. - Pour la détermination de tels modèles l'approche est assez comparable à la précédente. L'hypothèse de base la plus simple consiste à considérer un système à un seul compartiment représenté par le résidu et à supposer que la vitesse de diminution de la quantité de résidu $(\mathrm{dRt} / \mathrm{dt})$ est proportionnelle à celle qui est présente au même instant (Rt) :

$$
\mathrm{dRt} / \mathrm{dt}=-\mathrm{c} \cdot \mathrm{Rt} .
$$

Cette approche a été suggérée par Waldo, Smith et Cox (1969) et reprise plusieurs fois par la suite (Gill, Conrad et Hibbs, 1969; Smith et al., 1971 ; Mertens, 1973, etc...). Le tableau 1 regroupe les principaux développements proposés à partir de ce modèle de base. Les trois premiers supposent que la fraction dégradable est homogène $\left(1^{\text {er }}\right.$ ordre); ils tiennent compte ou non de l'existence d'une fraction non dégradable $(u)$ ou/et d'un temps de latence "discret 》 (to). Ils peuvent être combinés pour s'adapter à différentes situations : ainsi Mertens (1973), Dunlop et al. (1983), Nocek et English (1986), Robinson, Fadel et Tamminga (1986) ont montré l'intérêt d'un modèle à 6 paramètres du $1^{\text {er }}$ ordre avec deux fractions (Ro1, Ro2) et un temps de latence "discret " pour ajuster l'évolution des constituants pariétaux indigestibles:

$$
R t=R o 1 e^{-c 1(t-t o)}+R o 2 e^{-c 2(t-t o)}+u
$$

Le quatrième modèle du tableau 1 suppose que le taux de dégradation est nul pour $\mathrm{t}=\mathrm{o}$ et $\mathrm{s}^{\prime}$ accroît progressivement par la suite. Cette expression permet de tenir compte d'un temps de latence à évolution progressive et, de ce fait, d'une fraction dégradable non homogène. Sauvant et al. (1986) ont montré que ce modèle permettait d'ajuster plus précisément l'évolution des quantités de paroi cellulaire (NDF) non dégradées in sacco des aliments à paroi facilement digestible.

Robinson, Fadel et Tamminga (1986) ont également testé l'intérêt de modèles qui supposent que la dégradation est proportionnelle à la surface d'échange de l'aliment. L'expression la plus simple utilisée 
TABLEAU 1

Modèles d'ajustement de l'évolution des quantités résiduelles de substrat dans le réticulo-rumen.

\begin{tabular}{|c|c|}
\hline Hypothèse sous-jacente & Equation \\
\hline 1. Une fraction homogène totalement dégradable & $R t=R o \cdot e^{-c t}$ \\
\hline $\begin{array}{l}\text { 2. Une fraction homogène totalement dégradable associée à une } \\
\text { fraction non dégradable }(U)\end{array}$ & $\mathrm{Rt}=\mathrm{Ro}_{\mathrm{O}} \cdot \mathrm{e}^{-\mathrm{ct}}+\mathrm{U}$ \\
\hline 3. Intégration d'un temps de latence discret & $R t=R o \cdot e^{-c(t-t o)}+U$ \\
\hline 4. Intégration d'un temps de latence progressif & $R t=R_{0} \cdot e^{-c\left(1-e i_{i}\right)^{t}}+U$ \\
\hline
\end{tabular}

$\mathrm{Rt}=$ fraction résiduelle à l'instant $\mathrm{t} ; \mathrm{Ro}=$ fraction digestible présente à l'instant $\mathrm{t}=\mathrm{o} ; \mathrm{U}=$ fraction non dégradable $; \mathrm{c}=$ taux de dégradation de la fraction digestible $; \mathrm{h}=$ taux d'évolution de $\mathrm{c}$.

$$
R t=\left(\left(-\frac{k}{3}\right) t+R o^{1 / 3}\right)^{3}+u
$$

peut être complétée pour intégrer l'existence d'un temps de latence discret ou bien de plusieurs fractions distinctes par leur rythme de dégradation. Ces modèles n'ont pas permis d'améliorer l'ajustement des cinétiques mesurées pendant 50 jours de la quantité résiduelle de NDF de 5 aliments, par rapport à ceux du tableau 2 ou bien de l'équation (3).

\section{Les modèles agrégés.}

Ils ont pour but d'associer dans un même modèle plusieurs processus de la digestion ruminale.

L'agrégation des phénomènes de dégradation et de transit. - Pour estimer la proportion du substrat alimentaire théoriquement dégradée (DT) ou non dans le réticulo-rumen, il est nécessaire d'agréger les processus de dégradation et de transit. Cette démarche est appliquée dans plusieurs modèles globaux publiés depuis une dizaine d'années. L'agrégation se fait en supposant que dans un court intervalle de temps ( $\mathrm{dt}$ ) la quantité élémentaire de substrat disparaissant par dégradation $(\mathrm{dDt})$ est pondérée par la proportion de substrat encore présente à ce moment : $e^{-k t}$ ( $k$ étant le taux de renouvellement)

$$
d D t=(d D t / d t) \cdot e^{-k t} \cdot d t
$$

le calcul de la dégradabilité théorique (DTt) entre les temps o et t correspond donc à celui de l'intégrale

$$
\mathrm{DTt}=\int_{0}^{t}(d D t / d t) \cdot e^{-k t} \cdot d t
$$

Son calcul peut se faire pas à pas à partir des valeurs de dDt mesurées à des intervalles de temps successifs (Kristensen, Moller et Hvelplund, 1982). II est 
également possible, selon l'expression mathématique de (dDt/dt), d'obtenir une solution simple de l'intégration de DTt entre les temps o et infini. Ainsi, lorsque la cinétique de dégradation est décrite par l'équation (1), cette intégration aboutit à l'expression fréquemment utilisée à la suite de Orskov et McDonald (1979) :

$$
\mathrm{DT}=\mathrm{Dp} 1+\mathrm{Dp} 2\left(\frac{\mathrm{c}}{\mathrm{c}+\mathrm{k}}\right)
$$

Ces deux dernières méthodes ont été appliquées en supposant la valeur $\mathrm{k}=0,06 \mathrm{~h}^{-1}$, pour mettre au point la nouvelle version du système PDI (Vérité et al., 1987). D'autre part, par une approche comparable, Van Soest et al. (1982) ont proposé de calculer la DT de la fraction protéique (ou organique) d'un aliment par l'expression

$$
\mathrm{DT}=\sum_{1}^{n} \mathrm{pi}\left(\frac{\mathrm{ci}}{\mathrm{ci}+\mathrm{k}}\right)
$$

avec: $\mathrm{n}=$ nombre de fractions distinguées

pi $=$ proportion de la fraction $\mathrm{i}(\Sigma \mathrm{pi}=1)$

ci $=$ taux de dégradation de la fraction $\mathrm{i}$.

Cette expression peut être adaptée pour intégrer une phase de latence discrète en début de dégradation.

Waldo, Smith et Cox (1969) ont pris en compte l'influence du taux de transit $k$ de l'évolution des quantités résiduelles d'une fraction alimentaire dans le rumen en supposant qu'elle comporte 2 sous-fractions, l'une dégradable (RDt), à un taux $\mathrm{c}$, et l'autre non dégradable ( $\mathrm{RNt}$ ). Les équations différentielles de description simultanée

$$
\begin{aligned}
& \mathrm{dRDt} / \mathrm{dt}=-\mathrm{c} \cdot \mathrm{RDt}-\mathrm{k} \mathrm{RDt}=-(\mathrm{c}+\mathrm{k}) \mathrm{RDt} \\
& \mathrm{dRNt} / \mathrm{dt}=-\mathrm{k} \cdot \mathrm{RNt}
\end{aligned}
$$

permettent d'obtenir l'expression de l'évolution de la quantité de résidu présente à l'instant $t$

$$
R t=R D o \cdot e^{-(c+k) t}+R N o \cdot e^{-k t} .
$$

L'agrégation des phénomènes de dégradation, de transit et de croissance microbienne. - Sauvant et Giger (1987) ont proposé un modèle s'appuyant sur les cinétiques mesurées de dégradation des matières organiques (MO) et azotées des aliments pour calculer, compte tenu du transit, pour chaque aliment et différents intervalles de temps (0-1, 1-6,6-12,12-24, 24-48 h), les quantités de $\mathrm{MO}$ et $\mathrm{N}$ mises à disposition des microorganismes du rumen. Sur la base de ces informations, le modèle permet de calculer par programmation linéaire, des combinaisons d'aliments qui maximisent un critère, la protéosynthèse microbienne par exemple, tout en respectant un certain nombre de caractéristiques moyennes de valeur nutritive du régime. Cette approche permet de corriger le fait que l'évaluation de la protéosynthèse microbienne sur la base des quantités globales de matières azotées et organiques dégradées à l'aide des critères décrits ci-dessus n'est pas additive en raison des différences de "profil » des cinétiques de dégradation des constituants du régime. 
L'agrégation des phénomènes de dégradation et de réduction de la taille des particules et de transit. - Mertens et Ely (1979) ont élaboré un modèle de dégradation des parois cellulaires dans le rumen et le reste du tube digestif. $\mathrm{Ce}$ modèle, dont la structure de base est décrite à la figure 1, prend en compte 3 fractions de la paroi (indigestible, lentement ou rapidement digestible), ainsi que trois classes de taille de particules en fonction des résultats du tamisage sur 2 grilles ( 2 et $0,5 \mathrm{~mm}$ d'ouverture). Le système a 3 entrées, 9 compartiments ruminaux (fig. 1) et il est décrit par 12 équations différentielles dont 3 sont rapportées au tableau 2. La méthode de résolution de tels systèmes est évoquée plus loin. Ce modèle a été appliqué au cas de la digestion de la fraction NDF de fourrages.

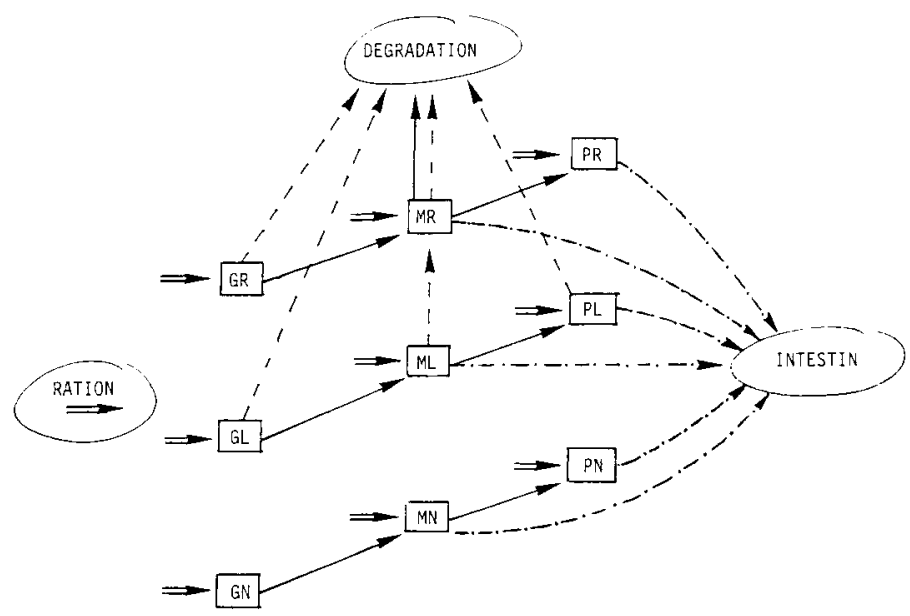

FIG. 1. - Diagramme du modèle de la digestion ruminale des parois cellulaires (Mertens et Ely, 1979). $(\mathrm{G}=$ grosses particules; $\mathrm{M}=$ particules movennes ; $\mathrm{P}=$ petites particules $; \mathrm{R}=$ rapidement dégradable; $\mathrm{L}=$ lentement dégradable $; \mathrm{N}=$ non dégradable.)

Les modèles d'encombrement du rumen. - Plusieurs auteurs ont tenté de modéliser les processus digestifs qui seraient susceptibles d'expliquer les variations de l'effet d'encombrement de la ration.

Mertens (1973) s'est appuyé sur l'équation (4) pour calculer un indice d'encombrement cumulé qui correspond au calcul des quantités de MS résiduelles dans le réticulo-rumen dues aux repas effectués dans les 240 h précédentes.

$$
\sum_{t=0}^{240}\left(\text { RDO } e^{-(c+k)(t-t 0)}+\text { RNO } \cdot e^{-k\left(t-t_{0}\right)}\right)
$$

Le pas de calcul est égal à 24/r, r étant le nombre de repas par jour. Ce modèle a permis de prévoir plus précisément la quantité de matière sèche volontairement 
TABLEAU 2

Equations différentielles de description de la digestion ruminale des parois cellulaires rapidement dégradables dans le modèle de Mertens et Ely (1979).

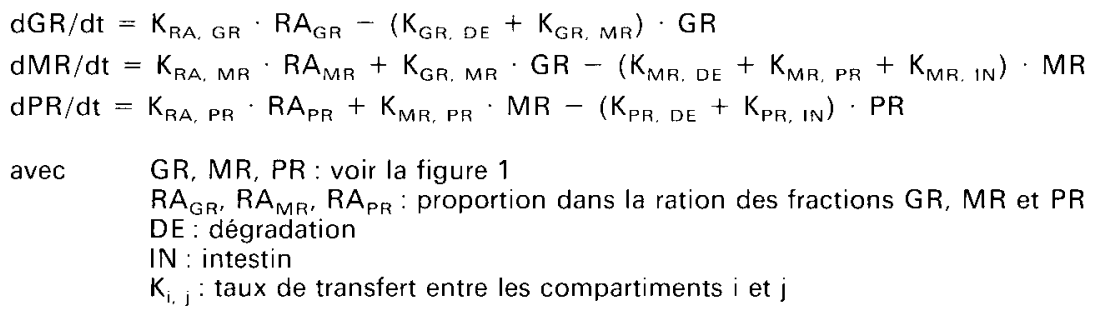

ingérée de 187 fourrages par le mouton. En outre, le paramétrage des différents coefficients avec des valeurs observées ont amené Mertens à conclure notamment que le taux de passage avait un rôle plus important sur l'indice d'encombrement que le taux de dégradation.

Bywater (1984) a proposé un modèle d'estimation de la quantité volontairement ingérée et de la digestion chez la vache laitière. Ce modèle comprend un sous-modèle rumen destiné à traduire le phénomène d'encombrement. Les flux ou informations d'entrée sont représentés par les principales caractéristiques de composition chimique, les proportions de fourrage et de concentré et la fréquence des repas. Des équations empiriques sont utilisées pour déterminer notamment la fraction digestible, la digestion et le transit des particules.

\section{Les modèles globaux.}

Les progrès des connaissances sur les principaux maillons digestifs du rumen ont permis la conception de modèles mécanistes les intégrant pour traduire les principaux aspects de son fonctionnement global. Hungate (1966) a réalisé une approche comparative systématique entre les propriétés du rumen et celles d'un appareil de type chemostat qui préfigurait la mise en place d'un modèle global. La première proposition concrétisée dans ce sens a été celle de Baldwin, Lucas et Cabrera (1970).

Dans la plupart des cas, le principe de base de l'élaboration du modèle consiste, après avoir déterminé les $n$ variables d'état $(X i)$ permettant de décrire les caractéristiques de l'écosystème ruminal, à définir un ensemble d'équations différentielles traduisant les variations élémentaires de ces variables d'état pendant un court intervalle de temps $\mathrm{dt}$.

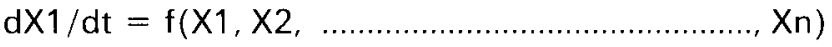

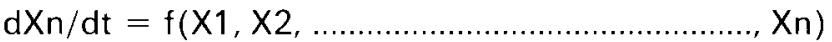

Dans les fonctions $f$ des seconds membres, les variables d'état n'interviennent généralement pas de façon linéaire, ce qui complique la résolution de tels 
systèmes. D'autres variables peuvent être prises en compte dans ces équations pour intégrer l'influence de facteurs exogènes (variables motrices) ou pour calculer des combinaisons de variables d'état présentant une signification pratique (variables auxiliaires).

La résolution met en œuvre des méthodes capables de calculer, à partir d'un ensemble de valeurs initiales (Xoi) des variables d'état, la trajectoire d'évolution temporelle du système repéré par les valeurs des Xi (Jolivet, 1983).

a. Le modèle de Baldwin. Lucas et Cabrera (1970). - Ce modèle a eu pour objet d'intégrer l'essentiel des connaissances quantitatives sur la digestion ruminale au sein d'un ensemble de 45 équations stœchiométriques du type général

$$
A+B \underset{k 1}{\leftrightarrows} C+D
$$

Dans cette expression A, B, C, D sont des produits, k1 et k2, les constantes de taux des réactions.

Ces équations décrivent en particulier:

- la dégradation des substrats et les produits formés (AGV, ATP, NADPH, $\mathrm{CH}_{4}$, $\mathrm{CO}_{2}$, chaleur) ;

- les besoins de croissance et les particularités métaboliques de plusieurs sous-populations microbiennes (cellulolytique, amylolytique, méthanogène) ; - l'absorption des nutriments azotés (pour intégrer le recyclage d'N).

A partir des 45 équations stœchiométriques établies, des équations différentielles exprimant le principe de la loi d'action de masse ont été élaborées pour définir l'évolution des concentrations (·) des différents produits, par exemple pour A :

$$
d(A) / d t=-k 1 \cdot(A) \cdot(B)+k 2 \cdot(C) \cdot(D)
$$

Ces équations ont concerné des substrats digestibles et trois sous-populations microbiennes présentant des profils fermentaires différents.

Ce modèle a été validé à travers différents contextes pratiques et a fourni des résultats jugés comme satisfaisants. Il possède cependant des limites importantes, en particulier, les différents substrats considérés sont supposés être totalement digestibles; en outre, il n'intègre pas de phénomène de transit et de régulation des processus digestifs (rumination...). D'autre part, l'évolution dynamique du système est permise par une variation des taux des réactions; cependant, la méthode mise en œuvre pour les déterminer n’est pas explicitée.

b. Le modèle de Reich/ et Baldwin (1975 et 1976). - L'originalité de cette approche est d'avoir recherché un modèle dit « équilibré », c'est-à-dire respectant l'égalité entre les flux d'éléments chimiques $(\mathrm{C}, \mathrm{H}, \mathrm{O}, \mathrm{N})$ entrant et sortant du 
rumen. Pour cela, le métabolisme ruminal est décrit par deux ensembles d'équations :

- des équations stœchiométriques pour les voies métaboliques principales ( $c f$. le modèle précédent) ;

- des équations d'égalisation des flux entrant et sortant des quatre éléments chimiques.

Une autre originalité de ce modèle est d'avoir fait appel à une résolution par programmation linéaire, ce qui permet d'une part d'exprimer les équations évoquées sous forme de contrainte et d'autre part de maximiser une fonction globale, l'utilisation des substrats par exemple. Ce modèle s'est montré peu fiable à partir du moment où l'alimentation était supposée être discontinue ou bien Iorsque la ration comprenait des aliments concentrés. Ce modèle a également été appliqué pour simuler la digestion sous l'effet de différents paramètres : interactions entre aliments et entre sous-populations microbiennes, efficacité de la croissance microbienne...

La programmation linéaire n'a été appliquée depuis qu'à un modèle partiel de fonctionnement du rumen (Sauvant et Giger, 1987) ce qui est peu en raison de l'intérêt potentiel qu'elle présente.

c. Le modèle de Ulyatt, Baldwin et Koong (1976). - Ce modèle a pour finalité d'intégrer et de décrire le plus précisément possible les mécanismes qui déterminent les variations de la valeur nutritive des aliments. Les substrats entrants sont au nombre de 10. Une description synthétique du sous-modèle "cellulose», qui est le plus compliqué est faite par ces auteurs (fig. 2). Une originalité de ce sous-modèle est de distinguer 2 tailles de particules vis-à-vis de leur possibilité de passage dans la suite du tube digestif. Les particules de plus grande taille peuvent en outre rejoindre les autres sous l'effet de la rumination ou de la dégradation microbienne, cette dernière agit également sur les particules de petites tailles qui sont en outre soumises au phénomène de transit. Ce modèle a été validé avec des résultats plutôt encourageants sur des données expérimentales mesurées sur des moutons ingérant du foin de luzerne.

d. Le modèle de Baldwin, Koong et Ulyatt (1977). - Ce modèle se place dans la même finalité que le précédent. II est plus complet puisqu'il contient 14 sous-modèles et permet de simuler le devenir digestif de 12 constituants des aliments, les deux autres sous-modèles étant respectivement consacrés à la croissance microbienne et à différentes articulations de calcul permettant de comparer les résultats à ceux d'expérimentations. Le sous-modèle relatif à l'azote est en fait la reprise de la proposition de Mazanov et Nolan (1976).

Ce modèle a fourni des résultats jugés comme globalement satisfaisants vis-à-vis de la prise en compte de variations du niveau d'ingestion et de fréquence de repas d'un mouton ingérant du foin de luzerne. Par contre, sa validation a été moins probante pour traduire la régulation des phénomènes de transit ainsi que la prolifération microbienne en cas d'apport azoté insuffisant. 


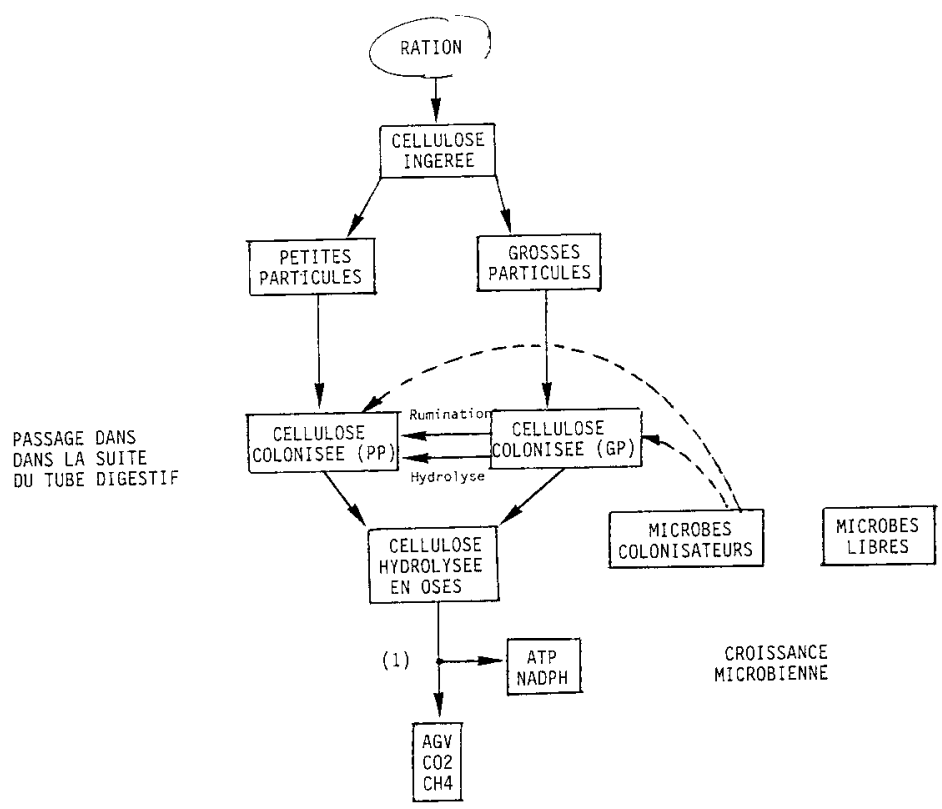

FIG. 2. - Diagramme de la sous-unité cellulose du modèle de Ulyatt, Baldwin et Koong (1976).

(1) Application des relations stœchiométriques de Baldwin, Lucas et Cabrera (1970).

Ce modèle a été ultérieurement repris par Baldwin et Denham (1979) pour simuler notamment l'influence du paramétrage du rythme de dégradation des protéines, de l'efficacité de la croissance microbienne.

e. Le modèle de Van Soest et al. (1980). - Ce modèle développe une première proposition de Fox et al. (1980). Par rapport aux premiers modèles statiques utilisés comme base des nouveaux systèmes d'alimentation azotée (Burroughs et al., 1975; Roy et al., 1977; Vérité et al., 1979) il présentait notamment l'originalité d'intégrer certaines composantes dynamiques et des possibilités de variation de la protéosynthèse microbienne, exprimée par rapport à la matière organique fermentescible (MOF), en fonction du renouvellement de la phase liquide $\left(0,02<\mathrm{KL}<0,20 \mathrm{~h}^{-1}\right)$ à partir de résultats publiés par Van Nevel et Demeyer (1979). En outre, 3 à 6 fractions protéiques alimentaires sont distinguées selon leur caractéristique de dégradation, et leur dégradabilité théorique est calculée à partir de l'équation (2). La quantité de MOF est prédite par une équation empirique à partir de la teneur en lignine des aliments.

Ce modèle est assez séduisant par sa simplicité ; il n'a cependant fait l'objet que d'un nombre très limité de validations expérimentales. II demande en outre à être plus largement «nourri» avec des données de base (caractéristiques d'aliments, valeurs des besoins) pour pouvoir déboucher sur un système opérationnel d'unités d'alimentation azotée. Fox, Sniffen et O'Connor (1987) et Sniffen et al. (1987) travaillent actuellement dans ce sens. 
f. Le modèle de Black et al. (1980-1981) et Beever et al. (1981). - Un groupe de chercheurs australiens a mis au point un modèle de description du métabolisme ruminal à partir d'informations concernant le niveau d'ingestion de matière sèche, la durée de rumination, la taille des particules du régime, 8 caractéristiques de composition du régime, le recyclage salivaire, les caractéristiques de dégradabilité potentielle des $\beta$-polyholosides et des protéines.

Ce modèle s'appuie largement sur des équations empiriques, ce qui le distingue nettement des autres propositions. II a pour objectif de décrire au mieux, par calcul itératif, la dynamique de production des protéines microbiennes et des différents produits terminaux. II a été validé par comparaison à des données expérimentales et par étude de sa stabilité lors de variations de la dégradabilité des protéines et des polyholosides du renouvellement de la phase aqueuse. Ce modèle sert de base au système australien d'unités d'alimentation azotée.

g. Le modèle de France, Thornley et Beever (1982). - Ce modèle comprend 9 compartiments, 7 fractions alimentaires ou substrats, les 2 autres correspondant aux microorganismes et au volume ruminal. La figure 3 rapporte la structure du système considéré pour permettre de préciser le principe de l'élaboration des 9 équations différentielles de description de son évolution (tabl. 3).

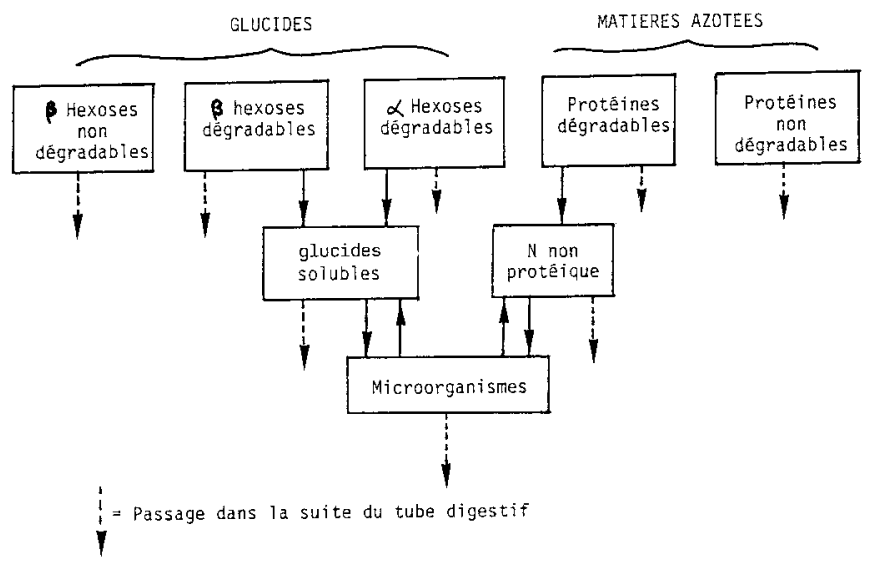

FIG. 3. - Structure du système ruminal (Modèle de France, Thornley et Beever, 1982).

(1) Le $9^{e}$ compartiment est représenté par le volume métabolique ruminal.

Dans ce travail, la croissance des microorganismes est modélisée en s'appuyant sur le principe d'une réponse selon une loi de Michaelis-Menten aux concentrations en substrats disponibles glucidique et azoté, en outre, il a été tenu compte d'un processus de mortalité microbienne inversement proportionnel au taux de croissance (tabl. 4). 
Equation différentielle de description de la variation de la concentration ruminale d'un constituant de la ration (France, Thornley et Beever. 1982).

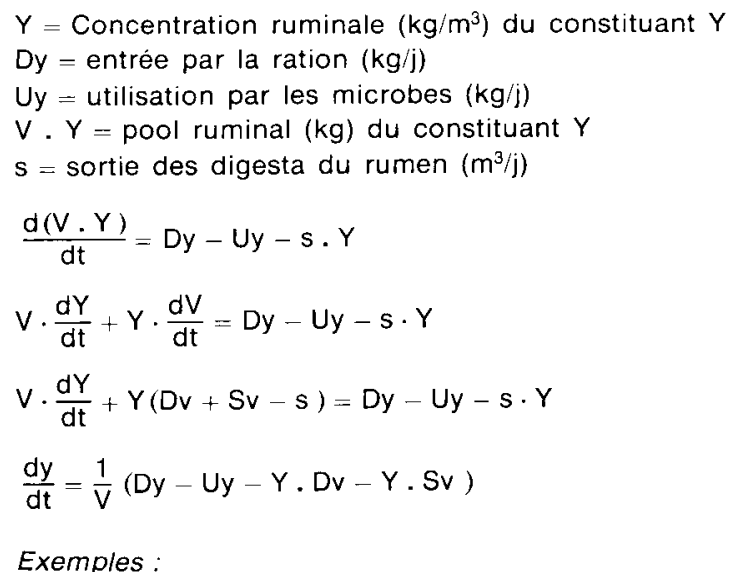

1) Constituant non dégradable $\Leftrightarrow U y=0$

Volume ruminal constant

$\frac{d Y}{d t}=\frac{1}{V}(D y-U y-s \cdot Y)$

2) Marqueur mis à $t_{0}$ à une concentration $Y_{0}(V=$ Constante $)$

$\frac{d y}{d t}=-\frac{s}{V} \cdot Y \Rightarrow Y=Y_{0} \cdot e^{-\frac{s t}{v}}$

$\frac{S}{v}=$ taux de dilution

3) Utilisation par les microbes proportionnelle à $Y, V,\left(\begin{array}{l}D y=0 \\ V=\text { constante }\end{array}\right)$

$\frac{d Y}{d t}=-k \cdot Y-\frac{s}{v} \cdot Y$

$Y=Y_{0} \cdot e^{-\frac{(k+s)}{v} t} \quad k=$ Taux de dégradation

h. Le modèle de Murphy, Baldwin et Ulyatt (1986). - Ce modèle constitue une actualisation des propositions $\mathrm{c}$ ) et surtout d) précédentes ; il en conserve la même structure globale en 14 sous-unités. Les modifications concernent en particulier l'intégration d'une durée de rumination (Murphy et al,, 1983), d'un compartiment particulaire supplémentaire et d'équations relatives à l'absorption des $A G V$ et à leur concentration dans le rumen.

Ce modèle a été validé sur plusieurs séries de résultats expérimentaux ; malgré des résultats globalement satisfaisants, il en ressort que des améliorations doivent 
TABLEAU 4

Modélisation de la multiplication microbienne dans le modèle de France. Thornley et Beever (1982),

1) Variations du taux de croissance $\mu$

$\mu=\mu m\left(1+\frac{k c}{C}+\frac{k n}{N}\right)^{-1}$

avec: $\mathrm{C}=$ Concentration en sucres solubles $\left(\mathrm{kg} / \mathrm{m}^{3}\right)$

$\mathrm{N}=$ Concentration en $\mathrm{N}$ non protéique $\left(\mathrm{kg} / \mathrm{m}^{3}\right)$

$\mu \mathrm{m}=$ Taux de croissance maximal

$\mathrm{kc}, \mathrm{kn}$ constantes de Michaelis

2) Croissance quotidienne : $\mathrm{g}(\mathrm{kg} / \mathrm{j})$

$g=\mu \cdot V \cdot M$.

avec $: V=$ Volume ruminal $\left(\mathrm{m}^{3}\right)$

$M=$ Matière sèche de la biomasse microbienne $\left(\mathrm{kg} / \mathrm{m}^{3}\right)$

3) Destruction microbienne

$\lambda=\lambda m(1+k \mu \cdot \mu)^{-1}$

$\lambda=\lambda \cdot V \cdot M$.

avec : $\lambda=$ taux de destruction

$\lambda \mathrm{m}=$ taux de destruction maximal

$k \mu=$ Constante de Michaelis

4) Variation de la biomasse microbienne

$d V \cdot M / d t=g-d-s \cdot M$

$d M / d t=\mu M-\lambda M-\frac{M}{V}(D v+S v)$

avec $: \mathrm{s}=$ Sortie des digesta

Dv = Entrée de liquide par l'alimentation

$\mathrm{Sv}=$ Entrée de salive

être recherchées sur la dynamique des particules et de la phase liquide et sur le métabolisme azoté.

i. Le modèle de Baldwin, Thornley et Beever (1987). - Ce modèle est le plus complet publié à ce jour ; il intègre l'acquis des différents modèles antérieurs et fait en outre lui-même partie d'un modèle général de fonctionnement du rumen appliqué à la vache laitière. L'objectif principal du modèle est la prédiction des quantités de nutriments absorbés par le tube digestif. II comprend 12 variables d'état relatives à des pools particulaires, à des substrats solubles ou non, aux microorganismes et à des produits des fermentations. Les différentes réactions sont supposées suivre des cinétiques conformes à la loi d'action de masse ou à la loi de Michaelis-Menten. Le système considéré est décrit par 17 équations différentielles. 
Ce modèle, qui a fait l'objet d'étude de stabilité au paramétrage des principales caractéristiques le déterminant, a été validé à travers les résultats expérimentaux de Sutton (1985). Malgré un bon comportement d'ensemble, il apparaît que ses relations stœchiométriques demandent à être revues, bien qu'elles correspondent à celles établies par Murphy, Baldwin et Koong (1982) à partir d'une large base de données bibliographiques. En outre, ces auteurs soulignent que leur modèle demande à être sérieusement précisé au niveau des pools de particules de tailles différentes (passage d'un pool à l'autre, taux de sortie du rumen, disponibilité pour les microorganismes).

\section{Discussion et conclusion.}

La modélisation de la digestion ruminale n'a pas pour objet de se substituer au travail expérimental de recherches. Par contre, elle sert de trait d'union entre deux grands objectifs : I'application, en particulier par sa contribution à la mise au point de systèmes d'unités d'alimentation, et la recherche, c'est-à-dire la détection des thématiques à approfondir.

Les modèles partiels, statiques ou dynamiques, proposés, s'appuient sur des systèmes peu complexes et sur des techniques mathématiques simples; dans la mesure où ils s'adressent à des événements majeurs de la digestion ruminale (dégradation, transit moyen) ils ont déjà largement permis de faire progresser les systèmes d'unités d'alimentation, dans le cas de l'azote en particulier.

Les modèles globaux correspondent plus à des modèles de recherches destinés à identifier par simulation les mécanismes pour lesquels les connaissances intégrées au modèle sont insuffisantes pour traduire assez fidèlement la réalité. Actuellement, les modèles élaborés butent en particulier sur la dynamique des particules et sur les variations des processus fermentaires en fonction des apports alimentaires concentrés. Pour les phénomènes de transit, il paraît nécessaire de prendre en compte un nombre de compartiments particulaires supérieur à 2 ou 3 et de mieux connaître le rôle des phénomènes masticatoires dans la comminution des particules. Les approches de Faichney et Poncet (non publiées) vont d'ailleurs dans cette direction puisqu'elles respectent mieux le fait que les particules sont réparties suivant un continuum de taille auquel on peut associer un gradient de rythmes d'écoulement hors du rumen. Un gros travail reste cependant nécessaire pour préciser par exemple la contribution des particules à la dynamique microbienne en fonction de leur taille. Pour ce qui concerne les variations des processus fermentaires, il conviendrait peut-être de chercher à modéliser plus précisément les phénomènes d'interaction digestive qui ont été jusqu'alors largement ignorés dans les différentes propositions; il faudrait en particulier établir un sous-modèle qui soit capable de traduire les variations du $\mathrm{pH}$ ruminal en fonction de ses principaux déterminants.

Une autre question importante est de savoir si la micropopulation ruminale peut être considérée comme un ensemble homogène ou non. Les tentatives de prise en compte de sous-populations (Baldwin, Lucas et Cabrera, 1970; Reichl 
et Baldwin, 1975) ont été plutôt décevantes, vraisemblablement parce que les sous-ensembles distingués étaient trop simplistes par rapport à la réalité. Cependant, les résultats de Jouany (1978) et de Demeyer et Van Nevel (1979) sur la défaunation, ceux de Murphy, Baldwin et Koong (1982) sur les variations des équations stœchiométriques en fonction du régime, et certains problèmes rencontrés pour valider les proportions d'AGV produits par les modèles les plus récents, plaident pour chercher à distinguer des sous-ensembles au sein de la micropopulation ruminale. Une approche expérimentale de ce problème est très délicate, voire utopique, en raison des difficultés à travailler sur des microorganismes anaérobies, de la multitude des espèces concernées et de leurs interactions. Les études d'interrelations entre 2 ou 3 groupes bien définis de microorganismes (cf. Gouet et al., 1986) constituent la voie d'approche la plus pratiquée pour comprendre, et peut-être modéliser au mieux le comportement de l'ensemble de la micropopulation ruminale.

C'est incontestablement dans le secteur de la digestion de l'azote que les modèles globaux de recherche et d'application sont les plus proches et ont pu être prolongés à travers des systèmes pratiques d'unités d'alimentation. Cette réussite doit servir d'exemple pour chercher à définir d'autres systèmes d'unités d'alimentation exprimées en flux de nutriments absorbés (acides gras volatils, glucose, acides gras).

Mis à part la proposition de Reichl et Baldwin (1975), les modèles les plus complexes proposés à ce jour présentent l'inconvénient commun de ne travailler que dans un seul sens, celui de la simulation des événements digestifs et des flux de nutriments absorbés à partir des caractéristiques de la ration. Or, il faudrait pouvoir disposer de modèles «réversibles » qui soient également capables de déterminer le régime alimentaire le plus adapté à un "profil » souhaité de nutriments absorbés. Cette réversibilité conditionne à l'évidence l'intérêt de la modélisation pour définir les futurs systèmes d'unité d'alimentation.

D'autres grandes questions n'ont pour ainsi dire pas été abordées à travers la modélisation de la digestion ruminale. Ainsi, bien que l'essentiel des facteurs explicatifs des variations des quantités ingérées se trouve au niveau du réticulo-rumen, les tentatives faites à ce niveau (Mertens, 1973; Bywater, 1984) n'ont pas fourni de résultats utilisables alors que dans ce secteur les plus gros progrès ont été obtenus avec les systèmes d'unité d'encombrement dans lesquels l'animal est considéré comme une «boîte noire " (Demarquilly et al., 1978; Jarrige et al., 1979 ; Kristensen, 1983 ; Dulphy et al., 1987). D'autre part, il est connu que le transit des particules est notamment en relation avec le niveau d'ingestion et la proportion de fourrages mais ces deux aspects essentiels sont ignorés des modèles globaux récents.

Les épreuves de validation des modèles globaux devraient être développées ; en effet, la démarche appliquée jusqu'alors consiste à comparer les résultats obtenus par le modèle à ceux d'une ou deux expériences. Cette méthode a une portée limitée dans la mesure où une expérimentation peut, pour de multiples raisons, aboutir à des résultats sensiblement éloignés d'une loi générale qui est susceptible d'apparaître par le regroupement d'un nombre suffisant d'expérimentations. En conséquence, il nous paraîtrait plus judicieux de ne valider les modèles 
qu'à travers des bases de données expérimentales confirmées et bien répertoriées. En outre, la validation des modèles les plus complexes, en particulier ceux qui s'appuient sur des systèmes d'équations différentielles à second membre non linéaire, doit être établie en collaboration avec des spécialistes de biométrie et de calcul numérique. En effet, ces modèles soulèvent des problèmes d'étude de sensibilité et d'analyse numérique (Tomassone, communication personnelle) qui sont quasiment passés sous silence dans les publications des modèles globaux.

Le développement des biofermenteurs est important dans les agro-industries et la modélisation de leur fonctionnement en vue d'optimiser leur pilotage fait également l'objet de recherches (Deshayes, 1980 ; Kolmberg et Ranta, 1982 ; Engasser, 1985). II serait donc souhaitable de pouvoir établir des liens avec ces recherches en vue de favoriser des transferts de méthodologie, chaque biofermenteur pouvant être en partie considéré comme un modèle de l'autre...

4 Journées sur la Nutrition et l'Alimentation des Herbivores, I.N.R.A., Paris, 24 et 25 mars 1988.

\section{Résumé.}

Les concepts de base de l'analyse systémique s'appliquent à l'écosystème ruminal. En raison, d'une part, de la complexité de sa structure et de son fonctionnement et, d'autre part, de son rôle déterminant dans la nutrition et l'alimentation des ruminants, le réticulo-rumen a été l'objet de travaux de modélisation. Les modèles proposés sont statiques ou dynamiques. Ces derniers ont connu le développement récent le plus important bien que les premiers aient largement contribué à faire progresser les connaissances. Le degré de complexité des modèles dynamiques est principalement proportionnel au nombre de phénomènes biologiques pris en compte. Les modèles simples décrivent par exemple un processus élémentaire de dégradation ou de transit, les modèles agrégés intègrent simultanément plusieurs processus tandis que les modèles globaux ont pour objectif de traduire l'essentiel du comportement de l'écosystème ruminal en intégrant les principaux maillons de son fonctionnement (modèles mécanistes). Ces derniers sont étudiés depuis une vingtaine d'années. Leur validation est en partie satisfaisante mais traduit une certaine incapacité de ces modèles à rendre compte fidèiement de certaines variations importantes liées au régime ou à la dynamique des particules ou des micropopulations.

\section{Références}

BALDWIN R. L., LUCAS H. L., CABRERA R., 1970. Energetic relationships in the formation and utilisation of fermentation end-products. A. T. PHILLIPSON, Physiology of digestion and metabolism in the ruminants. Oriel Press, pp. 319.

BALDWIN R. L., KOONG L. J., ULYATT M. J., 1977. A dynamic model of ruminant digestion for evaluation of factors affecting nutritive value. Agric. Systems, 2, 255-288.

BALDWIN R. L., KOONG L. J., 1979. Mathematical modelling in analyses of ruminant digestive function : phylosophy, methodology and application, 251-256. In Digestive physiology and metabolism in ruminants. Ed. Y. RUCKEBUSCH et J. P. THIVEND, MTP Press.

BALDWIN R. L., DENHAM S. C., 1979. Quantitative and dynamic aspects of nitrogen metabolism in the rumen : a modelling analysis. J. Anim. Sci., 49, 1631-1639.

BALDWIN R. L., THORNLEY J. H. M., BEEVER D. E., 1987. Metabolism of the lactating cow. 11 - Digestive elements of a mechanistic model. J. Dairy Res., 54, 107-131. 
BEEVER D. E., BLACK J. L., FAICHNEY G. J., 1981. Simulation of the effects of rumen function on the flow of nutrients from the stomach of sheep; part 2. Assessment of computer prediction. Agric. Systems, 6, 221-241.

BELAICH J.P., 1986. Le rendement de ta croissance et la biomasse active dans les biotopes anaérobies. Reprod. Nutr. Dévelop., 26, 129-145.

BLACK J. L., BEEVER D. E., FAICHNEY G. J., HOWARTH B. R., MCGRAHAM N., 1981 . Simulating of the effects of rumen function of the flow of nutrients from the stomach of sheep : description of a computer program. Agric. Systems, 6, 196-219.

BLAXTER K. L., McGRAHAM J. C., WAINMAN F. W., 1956. Some observations on the digestibility of food by sheep and on related problems. Br. J. Nutr., 10,69-91.

BYWATER A. C., 1984. A generalized model of feed intake and digestion in lactating cows. Agric. Systems, 13, 167-186.

BURROUGHS W., NELSON D. K., MERTENS D. R., 1975. Evaluation of protein nutrition by metabolizable protein and urea fermentation potential. J. Dairy Sci, 58,611-616.

CHENG K. J., COSTERTON J. W., 1979. Adherent rumen bacteria, their role in the digestion of plant material, urea and epithelial cells, 227-250. In Digestive physiology and metabolism in ruminants. Ed. Y. RUCKEBUSCH et P. THIVEND, MTP Press.

CRAIG W. M., BROWN D. R., BRODERICK G. A., RICKER D. B., 1984. Polysaccharide levels of fluid and particulate microbes. Can. J. Anim. Sci., 64, 62-63.

CZERKAWSKI J. W., 1984. Degradation of solid feeds in the rumen : spatial distribution of microbial activity and its consequences, 158-172. In Contro/ of digestion and metabolism in ruminants. Ed. L. P. MILLIGAN, W. L. GROVUM and A. DOBSON. Reston Book, Englewood Cliffs, N.J.

DEMARQUILLY C., ANDRIEU J., SAUVANT D., DULPHY J.P., 1978. Composition et valeur nutritive des aliments. In L'alimentation des ruminants. Ed. I.N.R.A. Publ., Route de St-Cyr, Versailles.

DEMEYER D., VAN NEVEL C., 1979. Effect of defaunation on the metabolism of rumen microorganisms. Br. J. Nutr., 42, 515-524.

DEMEYER D., VAN NEVEL C., 1986. Influence of substrate and microbial interaction of efficiency of microbial growth. Reprod. Nutr. Dévelop., 26, 161-179.

DESHAYES L., 1980 . Utilisation de modèles mathématiques pour l'optimisation en fermentation. Bull. Soc. Chim. Fr., 12, 24-34.

DHANOA M. S., SIDDONS R. C., FRANCE J., GALE D. L., 1985. A multicompartimental model to describe marker excretion patterns in ruminant faeces. Br. J. Nutr., 53, 663-671.

DULPHY J.P., FAVERDIN P., MICOL D., BOCQUIER F., 1987. Révision du système des unités d'encombrement. Bull. Tech., CRZV, Theix, I.N.R.A., 70, 35-48.

DUNLOP A. C., WADWORTH J. C., KALLAWAY R. C., WALLACE J. F., 1983. Fibre fermentation kinetics in the rumen of sheep. In Recent advance in animal nutrition in Australia. Univ. New England Publ., pp. 141-149.

DURAND D., 1983. La Systémique. Coll. Que sais-je? Ed. Presses Universitaires de France.

ELLIS W. C., MATIS J. H., LASCANO C., 1979. Quantitating ruminal turnover. Fed. Proc., 38, 2702-2706.

ELLIS W. C., MATIS J. H., POND K. R., MAHLOOJI M., 1984. Physical and chemical digestion of forage fragments with emphasis on stochatic heterogenous rate models, 34-42. In Modelling ruminant digestion and metabolism. Dept Anim. Sci., Univ. of California.

ENGASSER J. M., 1985. Modélisation physiologique des procédés de fermentation. Bio-sciences, 4, 127-131.

FAICHNEY G. J., 1984. The kinetics of particulate matter in the rumen, 173-195. In Control of digestion and metabolism in ruminants. Ed. L. P. MILLIGAN, W. L. GROVUM \& DOBSON. Reston Book, Englewood Cliffs, N.J.

FOX G. J., SNIFFEN C. J., VAN SOEST P. J., 1980. A net protein system for cattle: protein requirements of cattle. Symp. Oklahoma State Univ., $285 \mathrm{p}$.

FOX D. G., SNIFFEN C. J., O'CONNOR J. D., 1987. The Cornell net protein and carbohydrate system. I - Predicting cattle requirements, 36-41. In Proc. Cornell Nutrition Conference for feed manufacturers, Cornell Univ.

FRANCE J., THORNLEY H. M., BEEVER D. E., 1982. A mathematical model of the rumen. J. agric. Sci. Camb., 99, 343-353. 
GILL S. S., CONRAD H. R., HIBBS J. W., 1969. Relative rate of in vitro cellulose desappearance as a possible estimator of digestible dry matter intake. J. Dairy Sci. 52, 1687-1689.

GOUET P., GRAIN J., DUBOURGUIER H.C., ALBAGNAC G., 1986. Interactions entre espèces microbiennes anaérobies dans le rumen. Reprod. Nutr. Dévelop., 26, 147-159.

GROVUM W. L., WILLIAMS V. J., 1973. Rate of passage of digesta in sheep. 3 - Differential rates of passage of water and dry matter from the reticulo-rumen abomasum and caecum and proximal colon. Br. J. Nutr., 30, 231-239.

HUNGATE R. E., 1966. The rumen and its microbes. Acad. Press, New York.

JARRIGE R., JOURNET M., VÉRITÉ R., 1978. Chap. Azote, I.N.R.A., Alimentation des Ruminants. I.N.R.A.-Publications, Versailles, 89-128

JARRIGE R. et al., 1979. Le système des unités d'encombrement pour les bovins. Bull. Tech., CRZV. Theix, I.N.R.A., 38, 57-79.

JOLIVET E., 1983. Introduction aux modèles mathématiques en biologie. Actualités scientifiques et agronomiques $n^{\circ} 11$. Ed. I.N.R.A. et Masson, 151 p.

JOLIVET E., LAPLACE J.P., LEBAS F., 1975. Le transit digestif chez le lapin. IV - Essai de modélisation des transferts d'un marqueur radioactif entre les différents compartiments digestifs. Ann. Zootech., 24, 475-488.

JOUANY J. P., THIVEND P., 1972. Evolution post prandiale de la composition glucidique des corps microbiens du rumen en fonction de la nature des glucides du régime. I - Les protozoaires. Ann. Biol. anim. Bioch. Biophys., 12, 673-677.

JOUANY J. P., 1978. Contribution à l'étude des protozoaires ciliés du rumen : leur dynamique, leur rôle dans la digestion et leur intérêt pour le ruminant. Th. Doct. Univ. Clermont II, $195 \mathrm{p}$.

KOLMBERG A., RANTA J., 1982. Procedure for parameter and state estimation of microbial growth processes. Automatica, 18, 181-193.

KRISTENSEN E. S., MOLLER P. D., HVELPLUND T., 1982. Estimation of the effective protein degradability in the rumen of cows using the nylon bag technique combined with the outflow rate. Acta agric. scand., 32, 123-127.

KRISTENSEN E. S., 1983. Styring af foderoptagelsen ved hjaelp af foderrationens sammensaetning of valg af fodrings princip, Chap. 7, In Optimale foderrationer til malkekoen. Ed. Nat. Inst. Anim. Sci., Denmark.

McDONALD I., 1981. A revised model for the estimation of protein degradability in the rumen. $J$. agric. Sci. Camb., 96, 251-252.

MAZANOV A., NOLAN J. V., 1976. Simulation of dynamics of nitrogen metabolism in sheep. Brit. J. Nutr., 35, 149.

MEHREZ A. Z., ØRSKOV E. R., 1977. A study of the artificial fibre bag technique for determining the digestibility of feeds in the rumen. J. agric. Sci., 88, 645-650.

MERTENS D. R., 1973. Application of technical mathematical mode/s to cell wall digestion and forage intake in ruminants. Ph. D. Thesis, Cornell Univ. Agric., $217 \mathrm{p}$.

MERTENS D. R., ELY L. O., 1979. A dynamic model of fiber digestion and passage in the ruminant for evaluating forage quality. J. anim. Sci., 49, 1085-1089.

MERTENS D. R., 1982. Using neutral detergent fiber to formulate dairy rations. Proc. Georgia Nutr. Conf., p. 116.

MURPHY M. R., 1980. Modelling analyses of ruminant digestive function. Ph. D. Thesis, Univ. California, Davis.

MURPHY M. R., BALDWIN R. L., KOONG L. J., 1982. Estimation of stoichiometric parameters for rumen fermentation of roughage and concentrate diets. J. anim. Sci., 55, 411-421.

MURPHY M. R., BALDWIN R. L., ULYATT M. J., KOONG L. J., 1983. A quantitative analysis of rumination patterns. J. anim. Sci., 56, 1236-1240.

MURPHY M. R., BALDWIN R. L., ULYATT M. J., 1986. An uptake of a dynamic model of ruminant digestion. J. anim. Sci., 62, 1412-1422.

NOCEK J. E., ENGLISH J. E., 1986. In situ degradation kinetics: evaluation of rate determination procedure. J. Dairy Sci., 69, 77-87.

ØRSKOV E. R., MCDONALD I., 1979. The estimation of protein degradability in the rumen from incubation measurements weighted according to rate of passage. J. agric. Sci. Camb., 92, 499-503. 
OWEN F. N., GOETSCH A. L., 1984. Digesta passage and microbial protein synthesis, 196-223. In Contro/ of digestion and metabolism in ruminants. Ed. L. P. MILLIGAN, W. L. GROVUM, A. DOBSON, Ed. Reston Book, Englewood Cliffs N.J.

PIRT J., 1965. The maintenance energy of bacteria in growing cultures. Proc. roy. Soc. London, ser. B, 163, 224-231.

REICHL J. R., BALDWIN R. L., 1975. Rumen modelling: rumen input-ouput balanced models. J. Dairy Sci., 58, 879-890.

REICHL J. R., BALDWIN R. L., 1976. A rumen linear programming model for evaluation of concepts of rumen microbial function. J. Dairy Sci., 59, 439.

RICHARD F. J., 1959. A flexible growth function for empirical use. J. exper. Botany, 10, 290-300.

ROBINSON P. H., FADEL J.G., TAMMINGA S., 1986. Evaluation of mathematical models to describe neutral detergent residue in terms of its susceptibility to degradation in the rumen. Anim. Fd. Sci. Technol., 15, 249-271.

ROY J. H. B., BALCH C. C., MILLER E. L., ØRSKOV E. R., SMITH R. H., 1977. Calculation of the $\mathrm{N}$-requirements for ruminants from nitrogen metabolism studies, 126. In Protein metabolism and nutrition. Eur. Assoc. Anim. Prod. Pub. ${ }^{\circ} 22$, Wageningen.

SAUVANT D., BERTRAND D., GIGER S., 1985. Variations of the in sacco dry matter digestion of concentrates and by products. Anim. Feed. Sci. Technol., 13, 7-23.

SAUVANT D., DORLEANS M., DELACOUR C., BERTRAND D., GIGER S., $1986 . \quad$ La modélisation des cinétiques de dégradation des constituants pariétaux des aliments dans le rumen. Reprod. Nutr. Dévelop., 26, 303-304.

SAUVANT D., GIGER S., 1987. Straw digestibility calculation and digestive interactions. Proc. EEC Seminar COST $84^{\mathrm{b}}$, Evaluation of straw as ruminant feed, (in press).

SAUVANT D., 1988. Modelling of lactation and nutrition, 41-54. In Modelling of livestock production systems. EEC Seminar. Ed. S. KORVER, J. A. M. ARENDONK. Kluwer AC. Publ.

SMITH L. W., GOERING H. K., WALDO D. R., GORDON C. H., 1971. In vitro digestion rate of forage cell wall components. J. Dairy Sci, 54, 71-77

SNIFFEN C. J., FOX D. G., O'CONNOR J. D., VAN SOEST P. J., 1987. The Cornell net carbohydrate and protein system. II - Protein and carbohydrate partition in feeds and their utilisation, 42-47. In Proc. Cornell Nutrition Conference for feed manufacturers. Ed. Cornell Univ., USA.

SPEDDING C. R. W., 1988. General aspects of modelling and its application in livestock production, 3-9. In Modelling of livestock production systems. EEC Meeting. Ed. S. KORVER \& J. A. M. ARENDONK, Kluwer AC. Publ.

STOUTHAMER A. H., BETTENHAUSSEN C. W., 1973. Utilization of energy for growth and maintenance in continuous and batch cultures of microorganisms. A reevaluation of the method for the determination of ATP production by measuring molar growth yields. Biochem. biophys. Acta, 301, 53-70.

SUTTON J. D., 1985 . Digestion and absorption of energy substrates in the lactating cow. J. Dairy Sci. 68, 3376-3393.

TOMASSONE R., LESQUOY E., MILLIER C., 1983. La régression. Actualités scientifiques et agronomiques $n^{\circ} 13,180$ p. Ed. I.N.R.A. et Masson, Paris.

ULYATT M. J., BALDWIN R. L., KOONG L. J., 1976. The basis of nutritive value. A modelling approach. Proc. N.Z. Soc. anim. Prod., 36, 140-149.

VAN NEVEL C. J., DEMEYER D. I., 1979. Stoichiometry of carbohydrate fermentation and microbial growth efficiency in a continuous culture of mixed rumen bacteria. Eur. J. appl. Microbiol. Biotechnol., 7, 111-120.

VAN SOEST P. J., SNIFFEN C. J., MERTENS D. R., FOX D. G., ROBINSON P. H., KRISHNAMOORTHY U., 1980. P. 265-279. In Protein requirements for cattle. Symp. Oklahoma State Univ., $285 \mathrm{p}$

VÉRITÉ R., JOURNET M., JARRIGE R., 1979. A new system for the protein feeding of ruminants : the PDI system. Liv. Prod. Sci., 6, 349-367.

VÉRITÉ R., MICHALET-DOREAU B., CHAPOUTOT P., PEYRAUD J. L., PONCET C., 1987. Révision du système des protéines digestibles dans l'intestin (PDI). Bull. Tech. : CRZV-Theix, I.N.R.A., 70, 19-34.

VON BERTALANFFY L., 1979. Théorie générale des systèmes. Ed. Dunod, Paris. 
WALDO D. R., SMITH L. W., COX E. L., 1969. Model of cellulose disappearance from the rumen.J. Dairy Sci. 55, 125-129.

WALLISER B., 1977. Systèmes et modèles, 248 p. Ed. Leseuil, Paris. 